\title{
Propagation of nonclassical light through an absorbing and dispersive slab
}

\author{
M. Artoni ${ }^{1,2}$ and R. Loudon ${ }^{1}$ \\ ${ }^{1}$ Department of Physics, University of Essex, Colchester CO4 3SQ, England \\ ${ }^{2}$ Department of Physics and Applied Physics, University of Strathclyde, Glasgow G4 ONG, Scotland
}

(Received 23 July 1998)

\begin{abstract}
We calculate the effects of perpendicular propagation through a dispersive and absorbing dielectric slab at arbitrary temperatures on specific nonclassical properties of an incident light field. The transmitted signal is assumed to be measured by a detector that receives radiation only from the direction normal to the slab surfaces. Squeezing and nonclassical counting statistics of the transmitted light are evaluated for continuouswave squeezed states incident on both sides of the slab. The degree of second-order coherence is instead evaluated for an $\mathrm{N}$-photon incident pulse, and the effects of transmission through the slab on its antibunching are calculated. [S1050-2947(99)06701-3]
\end{abstract}

PACS number(s): 42.50.Dv, 12.20.- m, 42.50.Ar

\section{INTRODUCTION}

The electromagnetic field has recently been quantized for several sample geometries of a dispersive and absorbing dielectric material [1-5], and for wave propagation perpendicular to the sample surfaces. The quantization schemes are based on earlier work that is extensively reviewed in these references. We have applied the formalism to study the propagation of an optical pulse through a lossy and dispersive dielectric slab maintained at a finite temperature [6]. The optical properties of the pulse are modified by the temperature, dispersion, and absorption in the dielectric, and by the reflections from the slab surfaces. Some of these modifications, which are the same in classical and quantum treatments of pulse propagation, result in peak delays and shape distortions in comparison with the properties of the incident pulse, and these have been covered in our previous work [6]. However, for incident light of a nonclassical nature, there are also modifications of the quantum coherence and correlation properties of the pulse that can only be described by a quantum theory. Moreover, when the slab is maintained at a nonzero temperature, the quantum statistical features of the transmitted pulse are obscured or distorted by the addition of, or interference with, thermal emission. The aim of the present paper is to determine the effects of transmission and reflection by a thermal slab on specific nonclassical properties of the incident light such as quadrature squeezing, subPoissonian photon counting statistics, and antibunching.

The quantum treatment of propagation through an absorbing and dispersive thermal slab is briefly summarized in Sec. II. For incident light with nonclassical statistics, the main interest lies in the extent to which these are retained after transmission through the slab. A quantum theory for the optical detection of nonclassical features of light is not our intent here, but we use well-known results to derive general expressions for the photocount variance, Mandel $Q$ factor, and degree of second-order coherence of the transmitted field and then specialize them to useful closed-form expressions for specific situations of experimental interest. Thus the effects of propagation through the slab on initially squeezed light are derived in Sec. III and the effects on the Mandel $Q$ factor are determined in Sec. IV. The corresponding results for the antibunching of an incident $N$-photon pulse are ob- tained in Sec. V. In accordance with the one-dimensional nature of the field quantization, it is assumed throughout that the statistics of the transmitted light are measured by a photodetector whose field of view is restricted to the direction perpendicular to the slab surfaces. The conclusions of the work are discussed in Sec. VI.

\section{PRELIMINARIES}

The quantum-mechanical formalism for electromagnetic wave propagation through a lossy and dispersive dielectric slab [1-5] has been summarized previously [6]. We give here only the bare essentials needed for an appreciation of the present paper.

The form of the dielectric function for a slab of thickness $2 l$ is

$$
\varepsilon(x, \omega)=\left\{\begin{array}{l}
\varepsilon(\omega)=n^{2}(\omega)=[\eta(\omega)+i \kappa(\omega)]^{2} \text { for }|x| \leqslant l \\
1 \quad \text { for }|x|>l
\end{array}\right.
$$

where the complex refractive index $n(\omega)$ is assumed to be a known function, which is related to the real refractive index $\eta(\omega)$ and the extinction coefficient $\kappa(\omega)$, defined for positive frequencies. The real and imaginary parts of the dielectric function are related by the Kramers-Kronig relations and, in particular, the occurrence of absorption is an inevitable consequence of the presence of dispersion. The electromagnetic field is quantized on the basis of a continuous set of modes propagating in the direction perpendicular to the surfaces of the slab so that waves propagate parallel to the $x$ axis with their transverse-electric and magnetic-vector operators $\hat{\mathbf{E}}(x, t)$ and $\hat{\mathbf{B}}(x, t)$ aligned with the $y$ and $z$ axes, respectively. The schematic arrangement of the propagation geometry for the various components of the field is given in Fig. 1, which shows the notation for the slab input and output field operators.

The electric field operator $\hat{E}(x, t)$ at a detector in the region $x>l$ has the positive frequency component

$$
\begin{aligned}
\hat{E}^{(+)}(x, t)= & i \int_{0}^{\infty} d \omega \sqrt{\hbar \omega / 4 \pi \varepsilon_{0} c S}\left[\hat{b}_{R}(\omega) e^{i \omega x / c}\right. \\
& \left.+\hat{b}_{L}(\omega) e^{-i \omega x l c}\right] e^{-i \omega t}
\end{aligned}
$$




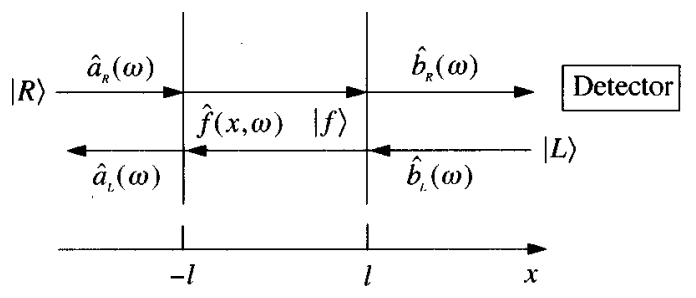

FIG. 1. Spatial configuration for the dielectric slab and notation for the destruction operators used in the definition of the relevant electric fields.

and the Hermitian conjugate expression for its negative frequency component. Here $S$ is the area of quantization in the $y z$ plane. The operator for the rightward-propagating outgoing field is given in terms of the leftwards and rightwards input fields by

$$
\hat{b}_{R}(\omega)=R(\omega) \hat{b}_{L}(\omega)+T(\omega) \hat{a}_{R}(\omega)+\hat{F}(\omega),
$$

where

$$
T(\omega)=4 n(\omega) e^{2 i \omega[n(\omega)-1] l / c} D^{-1}(\omega)
$$

and

$$
R(\omega)=e^{-2 i \omega l / c}\left[n^{2}(\omega)-1\right]\left[e^{4 i \omega n(\omega) l / c}-1\right] D^{-1}(\omega),
$$

with

$$
D(\omega)=[n(\omega)+1]^{2}-[n(\omega)-1]^{2} \exp [4 i \omega n(\omega) l / c],
$$

are the complex amplitudes of the transmission and reflection coefficients, respectively.

The operator $\hat{F}(\omega)$ represents the noise associated with the dissipation in the slab, and its form is given by

$$
\begin{aligned}
\hat{F}(\omega)= & i \sqrt{2 \omega \eta(\omega) \kappa(\omega) / c} \int_{-l}^{l} d x\left[V(\omega) e^{-i \omega n(\omega) x / c}\right. \\
& \left.+W(\omega) e^{i \omega n(\omega) x / c}\right] \hat{f}(x, \omega),
\end{aligned}
$$

where

$$
\begin{gathered}
V(\omega)=2[n(\omega)+1] \exp \{i \omega[n(\omega)-1] l / c\} D^{-1}(\omega), \\
W(\omega)=2[n(\omega)-1] \exp \{i \omega[3 n(\omega)-1] l / c\} D^{-1}(\omega),
\end{gathered}
$$

and the $\hat{f}(x, \omega)$ are spatially distributed Langevin noise current operators with the commutation relation

$$
\left[\hat{f}(x, \omega), \hat{f}^{\dagger}\left(x^{\prime}, \omega^{\prime}\right)\right]=\delta\left(x-x^{\prime}\right) \delta\left(\omega-\omega^{\prime}\right) .
$$

At finite temperature $\Theta$ the Langevin noise current operators have the expectation values

$$
\begin{aligned}
\langle f|\hat{f}(x, \omega)| f\rangle & =\left\langle f\left|\hat{f}^{\dagger}(x, \omega)\right| f\right\rangle=0, \\
\left\langle f\left|\hat{f}(x, \omega) \hat{f}\left(x^{\prime}, \omega^{\prime}\right)\right| f\right\rangle & =\left\langle f\left|\hat{f}^{\dagger}(x, \omega) \hat{f}^{\dagger}\left(x^{\prime}, \omega^{\prime}\right)\right| f\right\rangle=0,
\end{aligned}
$$

and

$$
\left\langle f\left|\hat{f}^{\dagger}(x, \omega) \hat{f}\left(x^{\prime}, \omega^{\prime}\right)\right| f\right\rangle=\bar{n}(\omega, \Theta) \delta\left(x-x^{\prime}\right) \delta\left(\omega-\omega^{\prime}\right),
$$

where

$$
\bar{n}(\omega, \Theta)=\left[e^{\hbar \omega / k \Theta}-1\right]^{-1}
$$

is the mean number of thermal photons at frequency $\omega$ and temperature $\Theta$. The $|f\rangle$ 's describe the states of a dissipative reservoir inside the slab, which are more formally represented by a statistical mixture.

All of the input and output operators in Eq. (2.3) satisfy boson commutation relations of the form

$$
\begin{aligned}
{\left[\hat{b}_{R}(\omega), \hat{b}_{R}^{\dagger}\left(\omega^{\prime}\right)\right] } & =\left[\hat{a}_{R}(\omega), \hat{a}_{R}^{\dagger}\left(\omega^{\prime}\right)\right]=\left[\hat{b}_{L}(\omega), \hat{b}_{L}^{\dagger}\left(\omega^{\prime}\right)\right] \\
& =\delta\left(\omega-\omega^{\prime}\right),
\end{aligned}
$$

and their consistency is ensured by the noise operator commutator

$$
\left[\hat{F}(\omega), \hat{F}^{\dagger}\left(\omega^{\prime}\right)\right]=\left[1-|R(\omega)|^{2}-|T(\omega)|^{2}\right] \delta\left(\omega-\omega^{\prime}\right),
$$

which is readily verified with the use of Eqs. (2.8)-(2.10).

\section{SQUEEZING}

In this section we examine how propagation through the slab influences the squeezing in an incident signal beam. Squeezing, specifically quadrature squeezing, occurs when the quantum fluctuations in one of the quadrature components of the electromagnetic field drops below the vacuum level: this is characterized by states of the field having negative normally ordered variances of the field operators, i.e., states with no classical analogues [7].

The effect of squeezing can be measured by a balanced homodyne detection scheme. For the field on the right-hand side of the slab (cf. Fig. 1) the difference between the integrated photocurrents in the two arms of a balanced detector can be represented by an operator of the form $[8,9]$

$$
\hat{O}=i \int_{t_{0}}^{t_{0}+T_{0}} d t\left\{\hat{b}_{R}^{\dagger}(t) \hat{a}_{\mathrm{LO}}(t)-\hat{b}_{R}(t) \hat{a}_{\mathrm{LO}}^{\dagger}(t)\right\}
$$

when the detector runs from time $t_{0}$ to time $t_{0}+T_{0}$. Here $\hat{a}_{\mathrm{LO}}(t)$ represents the field of the local oscillator, which is assumed to be in a coherent state $\left|\left\{\alpha_{\mathrm{LO}}\right\}\right\rangle$ with a single-mode amplitude of the form

$$
\alpha_{\mathrm{LO}}(t)=F_{\mathrm{LO}}^{1 / 2} \exp \left\{i \phi_{\mathrm{LO}}-i \omega_{\mathrm{LO}} t\right\} .
$$

Here $F_{\text {LO }}$ denotes the mean photon flux of the local oscillator, $\phi_{\mathrm{LO}}$ its phase, and $\omega_{\mathrm{LO}}$ its frequency. When the local oscillator is much more intense than the signal, the measurement operator (3.1) can be written in terms of a dimensionless homodyne electric field operator that characterizes the property of the signal measured at the detector,

$$
\hat{O}=\left(F_{\mathrm{LO}} T_{0}\right)^{1 / 2} \hat{E}\left(\phi_{\mathrm{LO}}, \omega_{\mathrm{LO}}\right),
$$

where 


$$
\begin{aligned}
\hat{E}\left(\phi_{\mathrm{LO}}, \omega_{\mathrm{LO}}\right)= & \frac{e^{i \phi_{\mathrm{LO}}}}{\sqrt{2 \pi T_{0}}} \int_{0}^{\infty} d \omega\left[\hat{b}_{R}^{\dagger}(\omega) e^{i\left(\omega-\omega_{\mathrm{LO}}\right) t_{0}}\right. \\
& \left.\times \zeta\left(\omega-\omega_{\mathrm{LO}}\right)+\text { H.c. }\right]
\end{aligned}
$$

and

$$
\zeta\left(\omega-\omega_{\mathrm{LO}}\right)=\frac{e^{i\left(\omega-\omega_{\mathrm{LO}}\right) T_{0}-1}}{\omega-\omega_{\mathrm{LO}}} .
$$

The variance of the field transmitted through to the right of the slab can then be written in the form

$$
\begin{aligned}
\left\langle\left[\Delta \hat{E}\left(\phi_{\mathrm{LO}}, \omega_{\mathrm{LO}}\right)\right]^{2}\right\rangle^{\text {out }} \\
=\frac{1}{2 \pi T_{0}} \int_{0}^{\infty} d \omega\left|\zeta\left(\omega-\omega_{\mathrm{LO}}\right)\right|^{2} \\
\quad+\frac{1}{\pi T_{0}} \int_{0}^{\infty} d \omega^{\prime} \int_{0}^{\infty} d \omega\left\{\left\langle\hat{b}_{R}^{\dagger}(\omega), \hat{b}_{R}\left(\omega^{\prime}\right)\right\rangle\right. \\
\quad \times \zeta\left(\omega-\omega_{\mathrm{LO}}\right) \zeta^{*}\left(\omega^{\prime}-\omega_{\mathrm{LO}}\right) e^{-i\left(\omega^{\prime}-\omega\right)\left(t_{0}+T_{0}\right)} \\
\quad-\operatorname{Re}\left[\left\langle\hat{b}_{R}^{\dagger}(\omega), \hat{b}_{R}^{\dagger}\left(\omega^{\prime}\right)\right\rangle e^{2 i \phi_{\mathrm{LO}} \zeta\left(\omega-\omega_{\mathrm{LO}}\right)}\right. \\
\left.\left.\quad \times \zeta\left(\omega^{\prime}-\omega_{\mathrm{LO}}\right) e^{i\left(\omega^{\prime}+\omega-2 \omega_{\mathrm{LO}}\right)\left(t_{0}+T_{0}\right)}\right]\right\}
\end{aligned}
$$

with the standard notation $\langle\hat{A}, \hat{B}\rangle \equiv\langle\hat{A}, \hat{B}\rangle-\langle\hat{A}\rangle\langle\hat{B}\rangle$ for a correlator. The homodyne electric field operator (3.4) has the useful property of a variance (3.6) equal to unity for the vacuum state. The amount of squeezing is conveniently gauged by the difference between the variance and unity.

The expectation values inside the integrals in Eq. (3.6) are over a product state that comprises the incident states of the field traveling leftwards $(|L\rangle)$ and rightwards $(|R\rangle)$ to the slab as well as states $|f\rangle$ that account for losses within the slab (cf. Fig. 1). We take $|L\rangle$ as a continuous-mode squeezed vacuum state, such as that produced by a degenerate parametric amplifier pumped at frequency $2 \Omega[8]$,

$$
|L\rangle=\hat{S}\left(\left\{\rho(\omega), \varphi_{\rho}(\omega)\right\}\right)|0\rangle \quad \text { (squeezed vacuum), }
$$

with

$$
\begin{aligned}
& \hat{S}\left(\left\{\rho(\omega), \varphi_{\rho}(\omega)\right\}\right) \\
& \quad=\exp \left\{\int d \omega \rho(\omega) e^{-i \varphi_{\rho}(\omega)} \hat{b}_{L}(\omega) \hat{b}_{L}(2 \Omega-\omega)-\text { H.c. }\right\},
\end{aligned}
$$

where $\varphi_{\rho}(\omega)$ is the phase and $\rho(\omega)$ is the strength characterizing the squeezed vacuum at a given frequency $\omega$. The state represented by Eq. (3.7) is a stationary light beam with a dimensionless mean photon $f l u x$ per unit angular frequency bandwidth given by $\sinh ^{2}[\rho(\omega)]$. The signal field traveling rightwards to the slab is taken as a squeezed coherent state with the same pump frequency $2 \Omega$,

$$
|R\rangle=\hat{D}(\{\alpha(\omega)\}) \hat{S}\left(\left\{\sigma(\omega), \varphi_{\sigma}(\omega)\right\}\right)|0\rangle
$$

(squeezed coherent state), with

$$
\hat{D}(\{\alpha(\omega)\})=\exp \left\{\int d \omega \alpha(\omega) \hat{a}_{R}^{\dagger}(\omega)-\text { H.c. }\right\},
$$

and analogous meanings for the parameters $\varphi_{\sigma}(\omega)$ and $\sigma(\omega)$. Here $\alpha(\omega)=|\alpha(\omega)| \exp \left[i \varphi_{\alpha}(\omega)\right]$ is the complex amplitude of the coherent component of the state $|R\rangle$, with a mean photon number per unit angular frequency bandwidth given by $|\alpha(\omega)|^{2}$. This choice of states enables one to investigate how the interference of the transmitted nonclassical signal field with a phase-sensitive vacuum, rather than the conventional one, affects the nonclassical statistics in the region of the detected fields. The expectation values in Eq. (3.6) are now evaluated with the use of Eq. (2.3) as

$$
\begin{aligned}
\left\langle\hat{b}_{R}^{\dagger}(\omega), \hat{b}_{R}\left(\omega^{\prime}\right)\right\rangle= & \delta\left(\omega-\omega^{\prime}\right)\left\{|R(\omega)|^{2} \sinh ^{2} \rho(\omega)\right. \\
& \left.+|T(\omega)|^{2} \sinh ^{2} \sigma(\omega)\right\} \\
& +\left\langle\hat{F}^{\dagger}(\omega) \hat{F}\left(\omega^{\prime}\right)\right\rangle, \\
\left\langle\hat{b}_{R}^{\dagger}(\omega), \hat{b}_{R}^{\dagger}\left(\omega^{\prime}\right)\right\rangle= & \frac{1}{2} \delta\left(\omega+\omega^{\prime}-2 \Omega\right) \\
& \times\left\{R^{* 2}(\omega) \sinh 2 \rho(\omega) e^{-i \varphi_{\rho}(\omega)}\right. \\
& \left.+T^{* 2}(\omega) \sinh 2 \sigma(\omega) e^{-i \varphi_{\sigma}(\omega)}\right\},
\end{aligned}
$$

which are independent of the incident coherent amplitude $\alpha(\omega)$. The noise in the slab originates from dissipation, described here by states $|f\rangle$ for which all expectation values are zero except those with an equal number of destruction and creation Langevin operators (Sec. II). From the definition (2.7) and the properties (2.10)-(2.14), the average flux of noise photons emitted by the thermal excitation of the slab is

$$
\begin{aligned}
\left\langle f\left|\hat{F}^{\dagger}(\omega) \hat{F}\left(\omega^{\prime}\right)\right| f\right\rangle= & \delta\left(\omega-\omega^{\prime}\right) 2 \bar{n}(\omega, \Theta)[\omega \eta(\omega) \kappa(\omega) / c] \\
& \times \int_{-l}^{l} d x \mid V(\omega) e^{-i \omega n(\omega) x / c} \\
& +\left.W(\omega) e^{+i \omega n(\omega) x / c}\right|^{2}
\end{aligned}
$$

that is conveniently expressed in terms of the reflection and transmission coefficients as

$$
\begin{aligned}
\left\langle f\left|\hat{F}^{\dagger}(\omega) \hat{F}\left(\omega^{\prime}\right)\right| f\right\rangle= & \bar{n}(\omega, \Theta)\left[1-|T(\omega)|^{2}-|R(\omega)|^{2}\right] \\
& \times \delta\left(\omega-\omega^{\prime}\right) .
\end{aligned}
$$

The noise flux clearly vanishes for a lossless slab, where $|R|^{2}+|T|^{2}=1$ at all frequencies.

The variance (3.6) depends on the time $T_{0}$ and, through Eqs. (3.11) and (3.12), it depends in a complicated fashion on the frequency dispersion of the incident squeezed fields (magnitude and phase) and on the specific form of the slab (complex) refractive index. Yet, a compact expression can be 
derived for sufficiently long $T_{0}$ 's (narrow detector bandwidth) in which case the products of the $\zeta$ 's in Eq. (3.6) tend to the delta function $\delta\left(\omega-\omega_{\mathrm{LO}}\right)$ when the local oscillator frequency is equal to the central frequency $\Omega$ of the squeezing. The measured field variance (3.6) simplifies to

$$
\begin{aligned}
& \left\langle\left[\Delta \hat{E}\left(\phi_{\mathrm{LO}}, \omega_{\mathrm{LO}}\right)\right]^{2}\right\rangle^{\text {out }}=1+2\left\{\bar{n}+|R|^{2}\left[\sinh ^{2} \rho-\bar{n}\right]\right. \\
& \left.+|T|^{2}\left[\sinh ^{2} \sigma-\bar{n}\right]\right\} \\
& -\operatorname{Re}\left[e ^ { 2 i \phi _ { \operatorname { L O } } } \left(R^{* 2} e^{-i \varphi_{\rho}} \sinh 2 \rho\right.\right. \\
& \left.\left.+T^{* 2} e^{-i \varphi_{\sigma}} \sinh 2 \sigma\right)\right] \text {, }
\end{aligned}
$$

where $\bar{n}$ is the mean number of thermal photons given by Eq. (2.14). For simplicity we have omitted writing the various functional dependencies from the coefficients on the righthand side, and the frequency-dependent functions are all evaluated at $\omega_{\mathrm{LO}}$. It is instructive to rewrite this result in terms of the variances of homodyne measurements made on the incident fields. For the field $|L\rangle$ traveling leftwards one has

$$
\begin{aligned}
\left\langle\left[\Delta \hat{E}\left(\phi_{\mathrm{LO}}, \omega_{\mathrm{LO}}\right)\right]^{2}\right\rangle_{|L\rangle}^{\mathrm{in}}= & 1+2 \sinh ^{2} \rho \\
& -\operatorname{Re}\left(e^{2 i \phi_{\mathrm{LO}}-i \varphi_{\rho}} \sinh 2 \rho\right) \\
= & e^{2 \rho} \sin ^{2}\left(\phi_{\mathrm{LO}}-\frac{1}{2} \phi_{\rho}\right) \\
& +e^{-2 \rho} \cos ^{2}\left(\phi_{\mathrm{LO}}-\frac{1}{2} \phi_{\rho}\right),
\end{aligned}
$$

and a similar expression for the rightwards-traveling incident field $|R\rangle$ obtains by replacing $\rho \rightarrow \sigma$ and $\phi_{\rho} \rightarrow \phi_{\sigma}$. Thus Eq. (3.15) can be written as

$$
\begin{aligned}
\left\langle\left[\Delta \hat{E}\left(\phi_{\mathrm{LO}}, \omega_{\mathrm{LO}}\right)\right]^{2}\right\rangle^{\text {out }}-1 \\
=2 \bar{n}\left(1-|R|^{2}-|T|^{2}\right) \\
\quad+|R|^{2}\left\{\left\langle\left[\Delta \hat{E}\left(\phi_{\mathrm{LO}}-\arg R, \omega_{\mathrm{LO}}\right)\right]^{2}\right\rangle_{|L\rangle}^{\mathrm{in}}-1\right\} \\
\quad+|T|^{2}\left\{\left\langle\left[\Delta \hat{E}\left(\phi_{\mathrm{LO}}-\arg T, \omega_{\mathrm{LO}}\right)\right]^{2}\right\rangle|R\rangle-1\right\},
\end{aligned}
$$

with the coefficients again evaluated at $\omega_{\mathrm{LO}}$. This result generalizes a squeezing conservation law previously derived for a lossless beam splitter [10] to the transmission and reflection at a lossy dielectric slab. We note that in the case of a slab at zero temperature $(\bar{n}=0)$ with an ordinary vacuum incident towards the left $(\rho=0)$, Eq. (3.15) can be written as

$$
\begin{aligned}
& \left\langle\left[\Delta \hat{E}\left(\phi_{\mathrm{LO}}, \omega_{\mathrm{LO}}\right)\right]^{2}\right\rangle^{\text {out }}-1 \\
& =|T|^{2}\left\{\left\langle\left[\Delta \hat{E}\left(\phi_{\mathrm{LO}}-\arg T, \omega_{\mathrm{LO}}\right)\right]^{2}\right\rangle|R\rangle=1\right\} .
\end{aligned}
$$

The scaling of the squeezing, as measured by the difference of the variance from unity, with the intensity transmission coefficient agrees with previous results $[11,12]$ for propagation through an absorbing slab. Jeffers and Barnett [13] have given a more detailed derivation of the effects of dispersion and frequency-dependent absorption on incident light with a Gaussian squeezing spectrum, and Schmidt, Knöll, and Welsch [14] have treated the propagation of squeezed light pulses, without inclusion of slab boundary effects.

We now use these results to analyze the propagation of quadrature noise through a thin film of a material whose
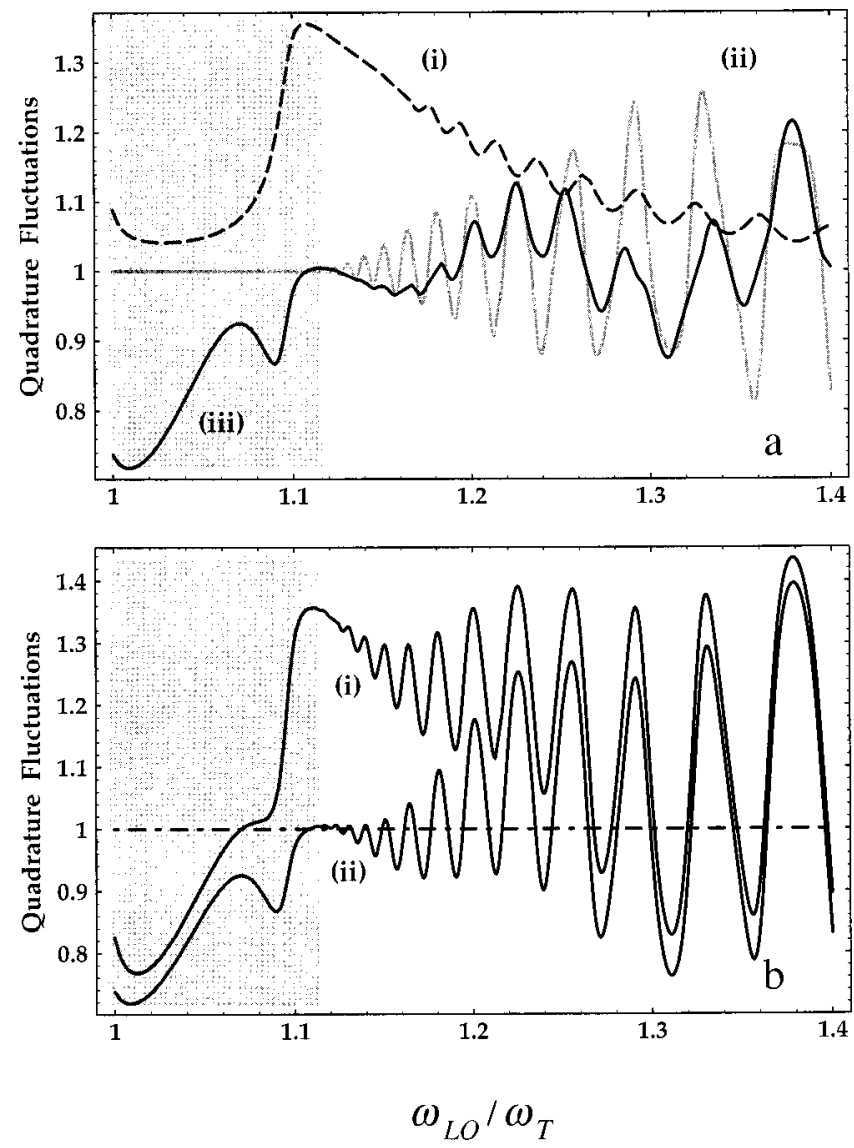

FIG. 2. Quadrature variance (3.15) of the field from a thin film as the local oscillator frequency $\left(\omega_{\mathrm{LO}}\right)$ is swept through the Reststrahl region, where $\varepsilon(\omega)$ is given by Eq. (3.19) with $\varepsilon(\infty)=8.5$, $\omega_{L}=1.11 \omega_{T}$, and $\Gamma=0.01 \omega_{T}$. The band-gap region is shaded. The film thickness $2 l$ and the transverse resonant frequency are such that $\omega_{T} l / c=10$. The incident squeezed vacuum state $|L\rangle$ has strength $\rho=0.2$ and phase $\varphi_{\rho}=2 \varphi_{\mathrm{LO}}-2$, while the squeezed coherent field $|R\rangle$ has the same strength $\sigma=0.2$ with $\varphi_{\sigma}=2 \varphi_{\mathrm{LO}}-5$. Part (a) shows the separate contributions from the thermal noise produced by the slab at temperature $\Theta=0.6 \hbar \omega_{T} / k$ (dashed curve i), the transmitted part of state $|R\rangle$ (gray curve ii), and the reflected part of state $|L\rangle$ (solid curve iii). The thermal contribution reduces to the unit vacuum-noise variance at $\Theta=0$. Part (b) shows the total noise for (i) $\Theta=0.6 \hbar \omega_{T} / k$ and (ii) $\Theta=0$. The input minimum noise levels are at 0.67 [cf. Eq. (3.13)].

dispersive and absorbing behaviors are described by a singleresonance complex permittivity $\varepsilon(\omega)$ of the form

$$
\varepsilon(\omega)=\varepsilon(\infty) \frac{\omega_{L}^{2}-\omega^{2}-i \omega \Gamma}{\omega_{T}^{2}-\omega^{2}-i \omega \Gamma}
$$

where $\omega_{T}$ and $\omega_{L}$ are the transverse and longitudinal frequencies of the resonance and $\Gamma$ is the damping. In Figs. 2 and 3 we examine the spectral features of the transmitted quadrature quantum noise for frequencies in the vicinity of the resonance when the squeezed vacuum $|L\rangle$ and the squeezed coherent field $|R\rangle$ have the same noise levels. The squeezing strengths $(\rho, \sigma)$ and phases $\left(\varphi_{\rho}, \varphi_{\sigma}\right)$ are taken to be constant over the resonance region. Parts (a) of the figures show the quadrature variances of each of the three contributions in the absence of the other two, and parts (b) show the 

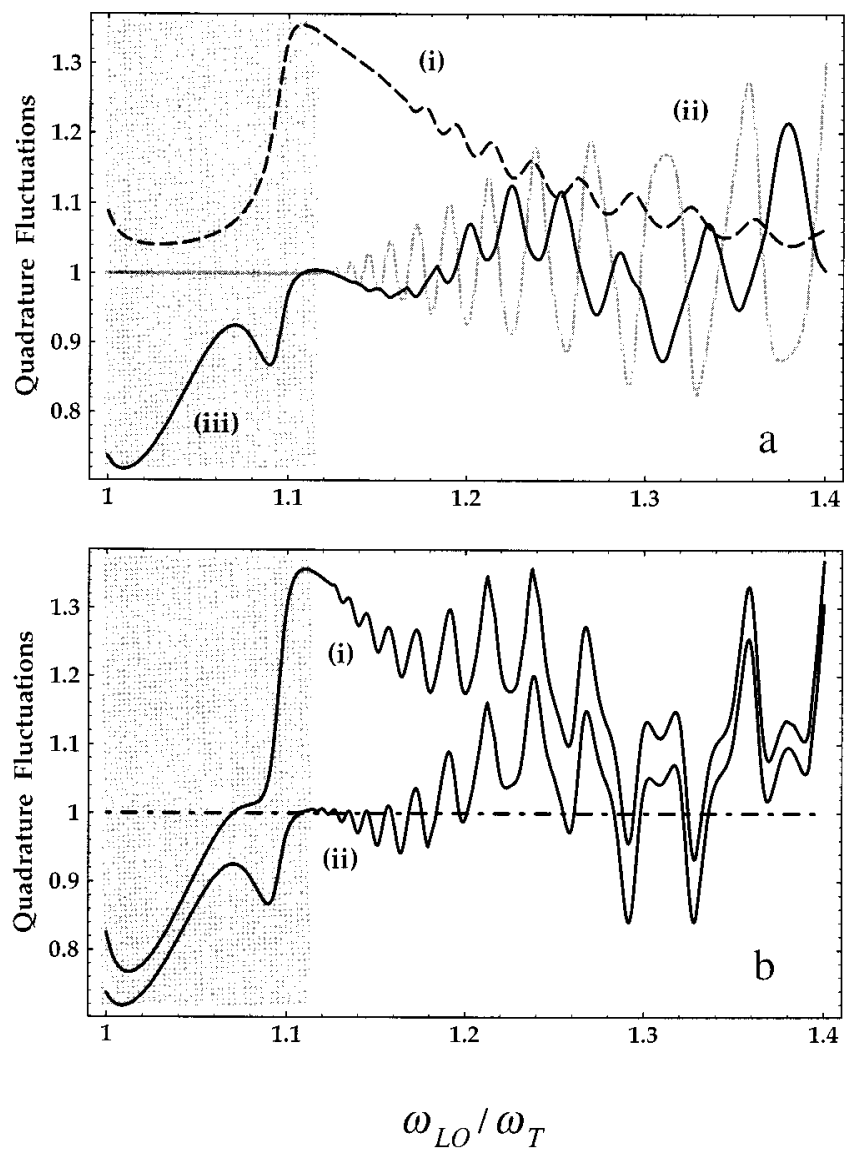

FIG. 3. Same as in Fig. 2 but with the different choice of phase $\varphi_{\sigma}=2 \varphi_{\mathrm{LO}}-2$.

combined variance, which equals the sum of the three contributions, less 2 to cancel the triple counting of the unit vacuum variance. The oscillatory behavior of the noise above the band gap is due to the phase of the complex reflection and transmission coefficients: the noise is sensitive not only to the local oscillator phase (fixed here) but to the relative phases $\phi_{\mathrm{LO}}-\arg R$ or $\phi_{\mathrm{LO}}-\arg T$, which are clearly dispersion dependent. A modest enhancement of the noise reduction with respect to the individual components arises from the coherent superposition of the reflected and transmitted noise, as is clearly seen by comparing Figs. 2 and 3. At $\Theta=0$, the thermal contribution is absent and maximum noise reduction occurs for frequencies within the band gap, where the transmission is small. At elevated temperatures the thermal noise reaches the maximum value of $2 \bar{n}$ around the band-gap edge, where reflection and transmission are both very small. The thermal noise diminishes on both sides of the edge, but faster inside than outside the gap because the increase of reflectivity for frequencies below $\omega_{L}$ is faster than the combined increase of reflectivity and transmissivity for frequencies above $\omega_{L}$. Moving further up above $\omega_{L}$ the thermal noise becomes vanishingly small because in our case $\hbar \omega_{T}$ is larger than $k \Theta$ and the combined values of the reflection and transmission keep increasing toward unity. The overall effect of the nonzero temperature is that of a substantial noise increase in the vicinity of the band-gap edge.

\section{THE PHOTON COUNTING STATISTICS}

In this section we examine how transmission through the slab affects the photon number distribution of an incident nonclassical light field. We analyze the counting statistics in terms of the deviation from classical (Poisson) statistics: the deviation is conveniently assessed in terms of the Mandel factor [15]

$$
Q \equiv \frac{\left\langle[\Delta \hat{N}]^{2}\right\rangle-\langle\hat{N}\rangle}{\langle\hat{N}\rangle}=\frac{\left\langle: \hat{N}^{2}:\right\rangle-\langle\hat{N}\rangle^{2}}{\langle\hat{N}\rangle},
$$

with the variance

$$
\left\langle[\Delta \hat{N}]^{2}\right\rangle=\left\langle\hat{N}^{2}\right\rangle-\langle\hat{N}\rangle^{2}
$$

and the normally ordered second moment

$$
\left\langle: \hat{N}^{2}:\right\rangle=\left\langle\hat{N}^{2}\right\rangle-\langle\hat{N}\rangle
$$

A vanishing $Q$ corresponds to coherent light, while a positive or negative $Q$ determines whether the photon-counting statistics are super-Poissonian $\left(\left\langle[\Delta \hat{N}]^{2}\right\rangle>\langle\hat{N}\rangle\right)$ or subPoissonian $\left(\left\langle[\Delta \hat{N}]^{2}\right\rangle<\langle\hat{N}\rangle\right)$. Since $Q$ must be non-negative for a classical stochastic field, sub-Poissonian photon statistics are regarded as an essentially quantum feature of the field $[7,15]$.

The Mandel factor is typically measured by direct photocount detection, which now replaces the balanced homodyne detection considered in Sec. III. The results of sets of measurements in which the photocurrent is integrated over periods of time $T_{0}$ can be predicted by the use of the dimensionless number operator [8]

$$
\hat{N}=\int_{t_{0}}^{t_{0}+T_{0}} d t \hat{b}_{R}^{\dagger}(t) \hat{b}_{R}(t)
$$

which represents the number of photons that arrive at the detector between $t_{0}$ and $t_{0}+T_{0}$. In practice, photon counters are sensitive to a bundle of frequencies whose bandwidth is generally small compared to their midfrequency. We model this by introducing a filter function $H(\omega)$ in terms of which the detected field at time $t$ is given by

$$
\hat{b}_{R}(t)=\frac{1}{\sqrt{2 \pi}} \int_{0}^{\infty} d \omega e^{-i \omega t} H(\omega) \hat{b}_{R}(\omega) .
$$

Using the input-output transformation (2.3) and the commutation relations (2.15), the expectation values needed for the Mandel factor read as follows:

$$
\begin{aligned}
\langle\hat{N}\rangle= & \frac{1}{2 \pi} \int_{t_{0}}^{t_{0}+T_{0}} d t \int_{0}^{\infty} d \omega_{1} \int_{0}^{\infty} d \omega_{2} e^{i\left(\omega_{1}-\omega_{2}\right) t} \\
& \times H^{*}\left(\omega_{1}\right) H\left(\omega_{2}\right)\left\langle\hat{b}_{R}^{\dagger}\left(\omega_{1}\right) \hat{b}_{R}\left(\omega_{2}\right)\right\rangle
\end{aligned}
$$

and 


$$
\begin{aligned}
\left\langle: \hat{N}:^{2}\right\rangle= & \frac{1}{(2 \pi)^{2}} \int_{t_{0}}^{t_{0}+T_{0}} d t \int_{t_{0}}^{t_{0}+T_{0}} d t^{\prime} \int_{0}^{\infty} d \omega_{1} \int_{0}^{\infty} d \omega_{2} \\
& \times \int_{0}^{\infty} d \omega_{3} \int_{0}^{\infty} d \omega_{4} e^{i\left(\omega_{1}-\omega_{3}\right) t+i\left(\omega_{2}-\omega_{4}\right) t^{\prime}} \\
& \times H^{*}\left(\omega_{1}\right) H^{*}\left(\omega_{2}\right) H\left(\omega_{3}\right) H\left(\omega_{4}\right) \\
& \times\left\langle\hat{b}_{R}^{\dagger}\left(\omega_{1}\right) \hat{b}_{R}^{\dagger}\left(\omega_{2}\right) \hat{b}_{R}\left(\omega_{3}\right) \hat{b}_{R}\left(\omega_{4}\right)\right\rangle .
\end{aligned}
$$

The expectation values inside Eqs. (4.6) and (4.7) will be evaluated for the same choice of states as in Sec. III, i.e., with a squeezed vacuum state $|L\rangle$ and a squeezed coherent state $|R\rangle$ traveling leftwards and rightwards to the slab, respectively, and the same states $|f\rangle$ accounting for losses within the slab. The actual evaluation is rather lengthy and will be reported separately in the Appendix.

As for the photocount variance of the previous section, Eqs. (4.6) and (4.7) are complicated functions of the integration time, the filter and incident radiation characteristics, and the dielectric slab dispersive and absorptive characteristics. A compact expression can again be derived for a situation of practical interest. For a Gaussian filter with bandwidth $\sigma_{H}$ and central frequency $\omega_{H}$,

$$
H(\omega)=\frac{1}{\sqrt{2 \pi}} \exp \left[-\frac{\left(\omega-\omega_{H}\right)^{2}}{2 \sigma_{H}^{2}}\right],
$$

the integrals in $\langle\hat{N}\rangle$ and $\left\langle: \hat{N}:^{2}\right\rangle$ can be evaluated with the help of the field expectation values given in the Appendix for the specific states of interest here. The multiple-frequency integrals in Eqs. (4.6) and (4.7) can be carried out taking advantage of the fact that in most cases of experimental interest the filter bandwidth is much smaller than its midfrequency $\left(\sigma_{H} \ll \omega_{H}\right)$ so that $R(\omega), T(\omega), \rho(\omega), \sigma(\omega), \varphi_{\rho}(\omega)$, $\varphi_{\sigma}(\omega)$, and $\alpha(\omega)$ do not vary much over $\sigma_{H}$ and can then be taken equal to their values at $\omega_{H}$. Since the integration time is much shorter than the inverse of the filter bandwidth $\left(\sigma_{H} T_{0} \ll 1\right)$, the multiple-time integrals in Eqs. (4.6) and (4.7) can also be done. Consider first the detected photon numbers defined as in Eq. (4.6) but produced by each incident state $|L\rangle$ or $|R\rangle$ in the absence of the other; for a slab at zero temperature with vanishing noise flux, the results are, respectively,

$$
\langle\hat{N}\rangle_{|L\rangle}=\frac{\sigma_{H} T_{0} \sqrt{\pi}}{(2 \pi)^{2}}|R|^{2} \sinh ^{2} \rho
$$

and

$$
\langle\hat{N}\rangle_{|R\rangle}=\frac{\sigma_{H} T_{0} \sqrt{\pi}}{(2 \pi)^{2}}|T|^{2}\left(\sinh ^{2} \sigma+2 \sigma_{H} \sqrt{\pi}|\alpha|^{2}\right) .
$$

Both these expressions contain a prefactor, determined by the detection period and bandwidth, followed by the intensity reflection and transmission coefficients of the slab, respectively, and completed by factors that characterize the strengths of the incident beams. The coherent component in Eq. (4.10) gains an additional detection bandwidth factor associated with the different definitions of $\sinh ^{2} \sigma$ and $|\alpha|^{2}$ as measures of the strengths of the squeezed and coherent components of the state $|R\rangle$ [see the discussion following Eqs. (3.8) and (3.10)]. The corresponding individual expression for the detected mean number of thermal photons emitted by the slab at an elevated temperature is

$$
\langle\hat{N}\rangle_{\text {thermal }}=\frac{\sigma_{H} T_{0} \sqrt{\pi}}{(2 \pi)^{2}} \bar{n}\left(1-|T|^{2}-|R|^{2}\right),
$$

which is consistent with expressions given in [6]. One thus arrives at the following expressions for the detected photonnumber mean and variance:

$$
\langle\hat{N}\rangle=\langle\hat{N}\rangle_{|L\rangle}+\langle\hat{N}\rangle_{|R\rangle}+\langle\hat{N}\rangle_{\text {thermal }}
$$

and

$$
\begin{aligned}
\left\langle: \hat{N}^{2}:\right\rangle-\langle\hat{N}\rangle^{2}= & \frac{\left(\sigma_{H} T_{0}\right)^{2}}{16 \pi^{3}}\left(|R|^{4} \cosh 2 \rho \sinh ^{2} \rho+|T|^{4}\right. \\
& \times\left\{\cosh 2 \sigma \sinh ^{2} \sigma+2 \sigma_{H} \sqrt{\pi}|\alpha|^{2}\right. \\
& \left.\times\left[2 \sinh ^{2} \sigma+\sinh 2 \sigma \cos \left(2 \varphi_{\alpha}-\varphi_{\sigma}\right)\right]\right\} \\
& +2|T|^{2}|R|^{2} \sinh ^{2} \rho\left(\sinh ^{2} \sigma+2 \sigma_{H} \sqrt{\pi}|\alpha|^{2}\right) \\
& +\frac{1}{2} \sinh 2 \rho \operatorname{Re}\left\{T ^ { 2 } R ^ { * 2 } \left[\sinh 2 \sigma e^{i\left(\varphi_{\sigma}-\sigma_{\rho}\right)}\right.\right. \\
& \left.\left.+4 \sigma_{H} \sqrt{\pi}|\alpha|^{2} e^{i\left(2 \varphi_{\alpha}-\varphi_{\rho}\right)}\right]\right\} \\
& +2 \bar{n}\left[1-|T|^{2}-|R|^{2}\right]\left[|R|^{2} \sinh ^{2} \rho\right. \\
& \left.+|T|^{2}\left(\sinh ^{2} \sigma+2 \sigma_{H} \sqrt{\pi}|\alpha|^{2}\right)\right] \\
& \left.+\bar{n}^{2}\left[1-|T|^{2}-|R|^{2}\right]^{2}\right) .
\end{aligned}
$$

For simplicity we omit writing the various functional dependencies of the coefficients on the right-hand side of Eq. (4.13), where all frequency-dependent functions are evaluated at the central frequency $\omega_{H}$. The first two terms in Eq. (4.13) originate, respectively, from the reflected squeezed vacuum field $|L\rangle$ and the transmitted squeezed coherent field $|R\rangle$; the former yields typical super-Poissonian statistics [7] while the latter, for a suitable choice of $2 \sigma_{H} \sqrt{\pi}|\alpha|^{2}$ and phase $2 \varphi_{\alpha}-\varphi_{\sigma}$, may reduce the super-Poissonian contributions associated with the "vacua" on both sides of the slab. The mixed third and fourth terms of Eq. (4.13) are nonvanishing only when squeezing is present on the right-hand side of the slab: for appropriate values of the phase $2 \varphi_{\alpha}-\varphi_{\rho}$, this term may further contribute to the nonclassical character of the transmitted counting statistics. The mixed penultimate term involves the slab thermal noise and the two incident fields, while the last term, always positive, is a contribution from the thermal noise alone.

The values of $Q$ defined by Eq. (4.1) for the contributions of each incident field $|L\rangle$ or $|R\rangle$ in the absence of the other, and for a slab at zero temperature, are, respectively,

$$
Q_{|L\rangle}=\frac{\sigma_{H} T_{0}}{4 \pi \sqrt{\pi}}|R|^{2} \cosh 2 \rho,
$$

in agreement with the usual result for a squeezed vacuum state $\{$ Eq. (3.23) in Ref. [7]\} scaled by a detection prefactor and by the intensity transmission coefficient of the slab, and 


$$
Q_{|R\rangle}=\frac{\sigma_{H} T_{0}}{4 \pi \sqrt{\pi}}|T|^{2} \frac{\cosh 2 \sigma \sinh ^{2} \sigma+2 \sigma_{H} \sqrt{\pi}|\alpha|^{2}\left[2 \sinh ^{2} \sigma+\sinh 2 \sigma \cos \left(2 \varphi_{\alpha}-\varphi_{\sigma}\right)\right]}{\sinh ^{2} \sigma+2 \sigma_{H} \sqrt{\pi}|\alpha|^{2}} .
$$

The value of $Q$ for the thermally emitted field alone, in the absence of the incident fields $|L\rangle$ and $|R\rangle$, is

$$
Q_{\text {thermal }}=\frac{\sigma_{H} T_{0}}{4 \pi \sqrt{\pi}} \bar{n}\left[1-|T|^{2}-|R|^{2}\right] \text {. }
$$

The composite Mandel factor is easily obtained by substitution of Eqs. (4.12) and (4.13) into Eq. (4.1), and there is no need to write down the complete expression. Owing to higher-order correlations, this composite $Q$ cannot be written as a sum of the individual contributions (4.14)-(4.16), as was done for the lower-order quadrature variance in Eq. (3.17), and the mean detected photon number in Eq. (4.12).

Standard results for special cases follow from Eqs. (4.12) and (4.13). Thus, when the field $|L\rangle$ is in an ordinary vacuum state $(\rho=0)$,

$$
\begin{aligned}
Q= & \frac{\sigma_{H} T_{0}}{4 \pi \sqrt{\pi}}\left(\bar{n}^{2}\left(1-|R|^{2}\right)^{2}+|T|^{4}\right. \\
& \times\left\{\bar{n}^{2}+\sinh ^{2} \sigma(\cosh 2 \sigma-2 \bar{n})+2 \sigma_{H} \sqrt{\pi}|\alpha|^{2}\right. \\
& \left.\times\left[2 \sinh ^{2} \sigma+\sinh 2 \sigma \cos \left(2 \varphi_{\alpha}-\varphi_{\sigma}\right)-2 \bar{n}\right]\right\} \\
& \left.+2 \bar{n}|T|^{2}\left(1-|R|^{2}\right)\left(\sinh ^{2} \sigma+2 \sigma_{H} \sqrt{\pi}|\alpha|^{2}-\bar{n}\right)\right) \\
& \times\left\{|T|^{2}\left(\sinh ^{2} \sigma+2 \sigma_{H} \sqrt{\pi}|\alpha|^{2}\right)+\bar{n}\left(1-|R|^{2}-|T|^{2}\right)\right\}^{-1} .
\end{aligned}
$$

This reduces to $Q_{|R\rangle}$, given by Eq. (4.15), when the slab is at zero temperature $(\bar{n}=0)$, and it further reduces to $Q=0$ if $|R\rangle$ is also an ordinary vacuum state. If the slab is removed $(T=1)$ and the coherent contribution to the mean photon number is much larger than the squeezing contribution, Eq. (4.17) agrees with the well-known result [Eq. (3.26) of Ref. [7]] for the Mandel $Q$ factor of a squeezed coherent state in free space.

With the help of the results (4.12) and (4.13), we show in Fig. 4 the effects of loss, dispersion, and temperature on the photon counting statistics by transmitting sub-Poissonian $(|R\rangle)$ and reflecting super-Poissonian $(|L\rangle)$ signals through a resonant dielectric slab at finite temperature and having the complex permittivity (3.17). The strengths $\rho, \sigma$, and phases $\varphi_{\rho}, \varphi_{\sigma}$ are taken to be constant over the resonance region. At $\Theta=0$, for the band-gap frequencies, the counting statistics are super-Poissonian as they originate from the reflected squeezed vacuum $|L\rangle$ (vanishing transmission), while they become progressively sub-Poissonian for modes above the band gap owing either to an increasing transmission of the incident sub-Poissonian field $|R\rangle$ or to the "coherent" superposition with the reflected squeezed vacuum $|L\rangle$ [cf. curves (ii) and (iii) in Fig. 4(a)]. When $\Theta>0$ the thermal noise adds in with a maximum value of $\bar{n}$ around the band-gap edge, but otherwise decreases. The explanation for the decrease of the thermal noise on both sides of the band-gap edge is the same as the one provided in Fig. 2(a) (i) or Fig. 3(a) (i). Elevated temperatures have the overall effect of degrading the nonclassical features of the number distribution for signal modes detected around the band-gap edge region.

\section{ANTIBUNCHING}

In this section we study how the degree of second-order coherence

$$
\begin{aligned}
g^{(2)}(x, t, \tau) & \\
= & \frac{\left\langle\hat{E}^{(-)}(x, t) \hat{E}^{(-)}(x, t+\tau) \hat{E}^{(+)}(x, t+\tau) \hat{E}^{(+)}(x, t)\right\rangle}{\left\langle\hat{E}^{(-)}(x, t) \hat{E}^{(+)}(x, t)\right\rangle\left\langle\hat{E}^{(-)}(x, t+\tau) \hat{E}^{(+)}(x, t+\tau)\right\rangle}
\end{aligned}
$$

of a quantized electromagnetic field is modified by the passage through a dispersive and absorbing dielectric slab that is kept at some finite temperature. The intensity correlation (5.1) is proportional to the joint probability of detecting photons at two times $t$ and $t+\tau$ and is measured in a timeresolved correlation experiment by a coincidence photocount detector placed at $x$. The intensity correlation (5.1) determines the effect of bunching or antibunching, which occur when $g^{(2)}(\tau)<g^{(2)}(\tau=0)$ or $g^{(2)}(\tau)>g^{(2)}(\tau=0)$, respectively. Photon bunching is the tendency of photons to distribute themselves preferentially in clusters rather than at random, so that when light falls on the photodetector, more photon pairs are detected close together in time than further apart. Antibunching is the opposite effect, in which fewer photon pairs are detected close together than further apart.

Unlike in the previous sections, we take the incident field $|L\rangle$ traveling towards the left to be in a conventional vacuum state $|0\rangle$, while the field $|R\rangle$ traveling towards the right is in a continuum photon-number state, denoted by $|N, \xi\rangle$. Such a state can be generated with the use of a quantum operator acting on the vacuum of the form [8]

$$
|R\rangle=|N, \xi\rangle=\frac{1}{\sqrt{N !}}\left[\int_{0}^{\infty} d \omega \xi^{*}(\omega) \hat{a}_{R}^{\dagger}(\omega)\right]^{N}|0\rangle .
$$

The function $\xi(\omega)$ describes the frequency distribution of the photon-number wave packet, whose form is determined by the way in which the photon state is prepared. In contrast to the stationary incident squeezed states assumed in Secs. III and IV, the number state $|N, \xi\rangle$ is taken in the form of an optical pulse. Single-photon [16] and two-photon [17] states with Gaussian wave packets, e.g., can be realized experimentally. We consider here a wave packet with a Gaussian distribution centered on $\omega_{c}$ (carrier) and a mean-square spatial length $L^{2}$,

$$
\xi(\omega)=\left(\frac{L^{2}}{2 \pi c^{2}}\right)^{1 / 4} \exp \left[-L^{2}\left(\omega-\omega_{c}\right)^{2} / 4 c^{2}\right],
$$



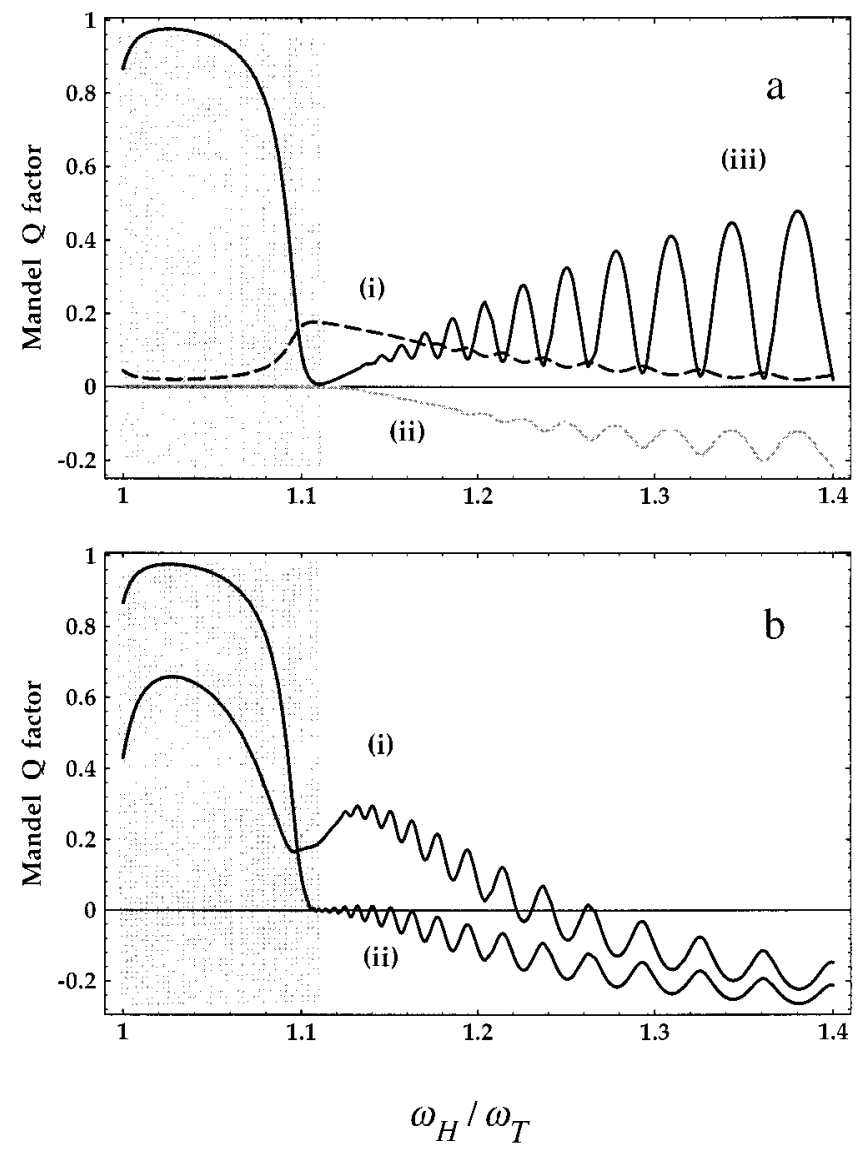

FIG. 4. Mandel $Q$ factor (4.1) of the field transmitted through dielectric slab of Fig. 2, in units of $\sigma_{H} T_{0} / 4 \pi \sqrt{\pi}$, as the midfrequency $\left(\omega_{H}\right)$ of a narrow-band photodetctor varies in the Reststrahl region. The incident field $|L\rangle$ is a squeezed vacuum $\left(\rho=0.2, \varphi_{\rho}\right.$ $=2 \varphi_{\alpha}$ ) with $Q=1.08$ (super-Poissonian). The incident field $|R\rangle$ is a squeezed coherent state $\left(\sigma=0.2, \varphi_{\sigma}=2 \varphi_{\alpha}-\pi, 2 \sigma_{H} \sqrt{\pi}|\alpha|^{2}=25\right)$ with $Q=-0.33$ (sub-Poissonian). Part (a) shows separate Mandel $Q$ factors from Eqs. (4.14)-(4.16) for the thermal noise of the slab at temperature $\Theta=0.6 \hbar \omega_{T} / k$ (dashed curve i), the transmitted part of the field $|R\rangle$ (gray curve ii), and the reflected part of the field $|L\rangle$ (solid curve iii). Part (b) shows the total $Q$ factor for (i) $\Theta$ $=0.6 \hbar \omega_{T} / k$ and (ii) $\Theta=0$.

The frequency spread of the wave packet is $c / L$ and its temporal length is of order $L / c$. For number states the reference value of Eq. (5.1), from which bunching or antibunching is assessed, is

$$
g_{N}^{(2)}=1-\frac{1}{N}
$$

independent of the form of the wave packet and the measurement position and times.

With this choice of states the transmitted intensity at $x$ $>l$ is

$$
\begin{aligned}
\left\langle\hat{E}^{(-)}(x, t) \hat{E}^{(+)}(x, t)\right\rangle \\
=\frac{\hbar}{4 \pi \varepsilon_{0} c S} \int_{0}^{\infty} d \omega_{1} \omega_{1}^{1 / 2} \int_{0}^{\infty} d \omega_{2} \omega_{2}^{1 / 2} e^{i\left(\omega_{1}-\omega_{2}\right) t_{r}} \\
\quad \times\left\langle f\left|\left\langle 0\left|\left\langle R\left|\hat{b}_{R}^{\dagger}\left(\omega_{1}\right) \hat{b}_{R}\left(\omega_{2}\right)\right| R\right\rangle\right| 0\right\rangle\right| f\right\rangle
\end{aligned}
$$

where the field operator is given by Eq. (2.2) and $t_{r}=t$ $-x / c$. The expectation value in the integrand can be evaluated from Eq. (A3) where the average flux of thermal photons is given by Eq. (3.14) and the average flux of the rightwards incident photons can be directly evaluated from Eqs. (5.2) and (5.3). The intensity Eq. (5.5) for an $N$-photon Gaussian wave packet is then [6]

$$
\left\langle\hat{E}^{(-)}(x, t) \hat{E}^{(+)}(x, t)\right\rangle=N\left|J_{1}\left(t_{r}\right)\right|^{2}+J_{2}(0),
$$

with the notation

$$
J_{1}(q) \equiv \sqrt{\hbar / 4 \pi \varepsilon_{0} c S} \int_{0}^{\infty} d \omega e^{-i \omega q} \omega^{1 / 2} T(\omega) \xi(\omega)
$$

and

$$
\begin{aligned}
J_{2}(q) \equiv & \frac{\hbar}{4 \pi \varepsilon_{0} c S} \int_{0}^{\infty} d \omega e^{-i \omega q} \omega \bar{n}(\omega, \Theta) \\
& \times\left[1-|R(\omega)|^{2}-|T(\omega)|^{2}\right]
\end{aligned}
$$

where $q$ is a generic time parameter.

Similarly one obtains for the intensity-intensity correlation in the numerator of Eq. (5.1),

$$
\begin{aligned}
& \left\langle\hat{E}^{(-)}(x, t) \hat{E}^{(-)}(x, t+\tau) \hat{E}^{(+)}(x, t+\tau) \hat{E}^{(+)}(x, t)\right\rangle \\
& =\left(\frac{\hbar}{4 \pi \varepsilon_{0} c S}\right) \int_{0}^{\infty} d \omega_{1} \omega_{1}^{1 / 2} \int_{0}^{\infty} d \omega_{2} \omega_{2}^{1 / 2} \int_{0}^{\infty} d \omega_{3} \omega_{3}^{1 / 2} \\
& \quad \times \int_{0}^{\infty} d \omega_{4} \omega_{4}^{1 / 2} e^{i\left(\omega_{1}-\omega_{4}\right) t_{r}} e^{i\left(\omega_{2}-\omega_{3}\right)\left(t_{r}+\tau\right)} \\
& \quad \times\left\langle f\left|\left\langle 0\left|\left\langle R\left|\hat{b}_{R}^{\dagger}\left(\omega_{1}\right) \hat{b}_{R}^{\dagger}\left(\omega_{2}\right) \hat{b}_{R}\left(\omega_{3}\right) \hat{b}_{R}\left(\omega_{4}\right)\right| R\right\rangle\right| 0\right\rangle\right| f\right\rangle .
\end{aligned}
$$

The expectation value in the integrand is now evaluated from Eq. (A4) where the relevant slab noise and incident field averages are carried out in much the same way as done for those in Eq. (A3). Similarly to Eq. (5.6) we can cast, after much algebra, the numerator of Eq. (5.1) into the compact form,

$$
\begin{aligned}
\left\langle\hat{E}^{(-)}\right. & \left.(x, t) \hat{E}^{(-)}(x, t+\tau) \hat{E}^{(+)}(x, t+\tau) \hat{E}^{(+)}(x, t)\right\rangle \\
= & N(N-1)\left|J_{1}\left(t_{r}\right)\right|^{2}\left|J_{1}\left(t_{r}+\tau\right)\right|^{2}+N J_{2}(0)\left[\left|J_{1}\left(t_{r}\right)\right|^{2}\right. \\
& \left.+\left|J_{1}\left(t_{r}+\tau\right)\right|^{2}\right]+2 N \operatorname{Re}\left[J_{1}\left(t_{r}\right) J_{1}^{*}\left(t_{r}+\tau\right) J_{2}(\tau)\right] \\
& +\left|J_{2}(\tau)\right|^{2}+J_{2}(0)^{2}
\end{aligned}
$$

Gathering together Eqs. (5.6) and (5.10) we obtain the degree of second-order coherence in the region $x>l$ (cf Fig. 1) when the incident field $|L\rangle$ is a conventional vacuum and the field $|R\rangle$ is an $N$-photon Gaussian wave packet, 


$$
\begin{aligned}
g_{N}^{(2)}\left(t_{r}, \tau\right)= & \frac{N(N-1)\left|J_{1}\left(t_{r}\right)\right|^{2}\left|J_{1}\left(t_{r}+\tau\right)\right|^{2}+N J_{2}(0)\left[\left|J_{1}\left(t_{r}\right)\right|^{2}+\left|J_{1}\left(t_{r}+\tau\right)\right|^{2}\right]}{\left[N\left|J_{1}\left(t_{r}\right)\right|^{2}+J_{2}(0)\right]\left[N\left|J_{1}\left(t_{r}+\tau\right)\right|^{2}+J_{2}(0)\right]} \\
& +\frac{2 N \operatorname{Re}\left[J_{1}\left(t_{r}\right) J_{1}^{*}\left(t_{r}+\tau\right) J_{2}(\tau)\right]+\left|J_{2}(\tau)\right|^{2}+J_{2}(0)^{2}}{\left[N\left|J_{1}\left(t_{r}\right)\right|^{2}+J_{2}(0)\right]\left[N\left|J_{1}\left(t_{r}+\tau\right)\right|^{2}+J_{2}(0)\right]} .
\end{aligned}
$$

The effect of the temperature on $g_{N}^{(2)}$ is entirely contained in the function $J_{2}$, which vanishes for a slab at $\Theta=0$.

Some simple limiting cases are immediately apparent using simple properties of $J_{2}(q)$ that are derived in more detail later in the section. In the absence of an incident number state one has

$$
g_{0}^{2}(\tau)=1+\frac{\left|J_{2}(\tau)\right|^{2}}{J_{2}(0)^{2}} \quad(N=0)
$$

For zero time delay, $\tau=0$, this takes the value of 2 expected for the chaotic light radiated by a thermal slab, while for $\tau^{\prime}$ 's that are larger than the thermal light coherence time $\tau_{c}$ $=\hbar / k \Theta, g_{0}^{(2)}(\tau)$ tends to unity as $\left|J_{2}(\tau)\right|$ becomes much smaller than $J_{2}(0)$ (real). In the presence of an incident number state $(N \neq 0)$ and a slab at zero temperature $\left(J_{2}=0\right)$, the degree of second-order coherence (5.11) reduces to that given in Eq. (5.4); this degree of second-order coherence is less than unity and it remains unchanged as the pulse propagates through the slab. More generally for $\tau=0$, Eq. (5.11) reduces to

$$
g_{N}^{(2)}\left(t_{r}\right)=2-\frac{N(N+1)\left|J_{1}\left(t_{r}\right)\right|^{4}}{\left[N\left|J_{1}\left(t_{r}\right)\right|^{2}+J_{2}(0)\right]^{2}} \quad(\tau=0) .
$$

For low temperatures and large photon numbers, where $J_{2}(0) \ll N\left|J_{1}\left(t_{r}\right)\right|^{2}$ and the contribution from the pulse dominates that from the slab, this reduces to the value (5.4) but at high temperatures and small photon numbers, where $J_{2}(0)$ $\gg N\left|J_{1}\left(t_{r}\right)\right|^{2}$ and the slab contribution is dominant, the degree of second-order coherence (5.13) tends to the chaotic light value of 2. Apart from these limit cases, bunching or antibunching from the full form of the degree of secondorder coherence in Eq. (5.11) can be assessed only when explicit expressions for the integrals $J_{1}(q)$ and $J_{2}(q)$ are available. Their evaluation requires the inclusion of appropriate parts of the mode functions (2.4)-(2.6) along with a specific form of the (complex) refractive index of the medium. This makes the integration hard to perform in general and the subsequent results difficult to handle except for some situations of experimental relevance, which will be examined in the following.

We proceed to derive a particular closed-form expression for $J_{1}(q)$. We consider a pulse whose length $L$ is much greater than the slab thickness, or $L \gg 2 \ln (\omega) / \pi$, so that the transmitted pulse retains the appearance of a single pulse, without breaking up into a series of component pulses [18]. Under this condition the pulse frequency spread $c / L$ is much smaller than the oscillation frequency of $|T(\omega)|$. We assume that the carrier frequency $\omega_{c}$ coincides with one of the relative maxima of $T(\omega)$ which, to a very good approximation, can then be taken equal to its value $T_{c}$ at $\omega_{c}$ over the whole interval of integration. The real refractive index $\eta(\omega)$ and the extinction coefficient $\kappa(\omega)$ can also be taken to be constant over the narrow pulse bandwidth and exactly equal to their values $\eta_{c}$ and $\kappa_{c}$ at the carrier frequency. In addition, since the pulse frequency spread $c / L$ is generally much smaller than $\omega_{c}$, we replace the lower bound in the integral by $-\infty$ and the square-root frequency factor by $\sqrt{\omega_{c}}$. Given these simplifications the integral in Eq. (5.7) can be carried out to obtain

$$
J_{1}(q)=\sqrt{\hbar \omega_{c} / 4 \pi \varepsilon_{0} L S}(8 \pi)^{1 / 4} T_{c} e^{-i \omega_{c} q-(c q / L)^{2}} .
$$

The evaluation of Eq. (5.14) at the time when the peak of the pulse enters the detector then yields

$$
\left|J_{1}\left(t_{r}+\tau\right)\right|^{2}=\frac{\hbar \omega_{c}\left|T_{c}\right|^{2}}{L S \varepsilon_{0} \sqrt{2 \pi}} \exp \left\{-\frac{2 c^{2} \tau^{2}}{L^{2}+2 l^{2}\left(\eta_{c}^{2}-1\right)^{2}}\right\},
$$

which, in turn, provides an expression for $J_{1}\left(t_{r}\right)$ when $\tau$ is set equal to 0 . This expression accounts for modifications due to the slower travel time and multiple internal reflections inside the slab through the increase $2 l^{2}\left(\eta_{c}^{2}-1\right)^{2}$ of the pulse mean-square width. We neglect, on the other hand, pulse modifications due to dispersion and absorption, which are of less importance in this context and are fully treated in [6].

A closed-form expression for $J_{2}(q)$ will also be given. We take again $\eta(\omega) \rightarrow \eta_{c}$ and $\kappa(\omega) \rightarrow \kappa_{c}$ constant over the narrow bandwidth of the pulse. With the help of Eqs (2.4)(2.6), after some effort, it is possible to show that

$$
\begin{aligned}
J_{2}(q)= & \frac{\hbar c / \pi \varepsilon_{0} S l^{2}}{\left[\left(\eta_{c}+1\right)^{2}+\kappa_{c}^{2}\right]^{2}} \\
& \times\left\{\eta_{c}\left[\left(\eta_{c}+1\right)^{2}+\kappa_{c}^{2}\right] I_{0}(q, 0,0)\right. \\
& -4\left|n_{c}\right|^{2} I_{0}\left(q, 4 \kappa_{c}, 0\right)-\eta_{c}\left(\left|n_{c}\right|^{2}\right. \\
& \left.-2 \eta_{c}+1\right) I_{0}\left(q, 8 \kappa_{c}, 0\right)+i \kappa_{c}\left(\left|n_{c}\right|^{2}-1\right) \\
& \times\left[I_{0}\left(q, 4 \kappa_{c}, \eta_{c}\right)-I_{0}\left(q, 4 \kappa_{c},-\eta_{c}\right)\right] \\
& \left.+2 \kappa_{c}^{2}\left[I_{0}\left(q, 4 \kappa_{c},-4 \eta_{c}\right)+I_{0}\left(q, 4 \kappa_{c}, 4 \eta_{c}\right)\right]\right\},
\end{aligned}
$$

where

$$
I_{0}(q, a, b)=\left(\frac{l}{c}\right)^{2} \int_{0}^{\infty} d \omega \omega \frac{e^{-[a l-i(b l+q c)](\omega / c)}}{e^{\hbar \omega / k \Theta}-1} .
$$


An analytic expression for this integral exists in terms of special functions, but it is not useful. We find it convenient to change the integral into a converging sum whose evaluation is readily performed [19]. We rewrite the denominator in Eq. (5.17) in terms of a progressive series so that the resulting exponential can be straightforwardly integrated to obtain

$$
I_{0}(q, a, b)=\sum_{n=1}^{\infty} \frac{l^{2}}{[a l+i(b l+q c)+(n \hbar c / k \Theta)]^{2}} .
$$

The final form of the degree of second-order coherence is obtained by substituting Eqs. (5.15) and (5.16) into Eq. (5.11).

One observes from Eq. (5.15) that for large delays, $\left|J_{1}\left(t_{r}+\tau\right)\right|$ in Eq. (5.11) is very small, and the degree of second-order coherence becomes

$$
g_{N}^{(2)}\left(t_{r}, \tau \gg L / c\right) \rightarrow 1+\frac{\left|J_{2}(\tau)\right|^{2}}{J_{2}(0)\left[J_{2}(0)+N\left|J_{1}\left(t_{r}\right)\right|^{2}\right]} .
$$

For an incident pulse much broader than the thermal coherence length $c \tau_{c}$, which is typically the case for pulses of duration longer than a few picoseconds and a slab temperature higher than about $3 \mathrm{~K}$, the second term of Eq. (5.19) falls rapidly to zero, $\left|J_{2}(\tau)\right|$ being much smaller than $J_{2}(0)$ when $\tau \gg \tau_{c}$. The behavior of the degree of second-order coherence (5.11) for intermediate time delays $\tau$ is otherwise not easily assessed, and in Fig. 5 the function is plotted for single- and two-photon Gaussian pulses propagating through a slab with different temperatures and absorption parameters. At very low temperatures in part (a), the pulse contribution to $g_{1,2}^{(2)}$ is dominant and the transmitted signal retains its antibunched character to delays of the order of the pulse duration $L / c$. For longer time delays essentially only the uncorrelated vacuum will be detected producing a unit secondorder coherence. For the numerical values chosen in Fig. 5, at low temperatures $L / c$ is about two orders of magnitude longer than $\tau_{c}$. At room temperatures in part (b), the thermal contribution is instead dominant and the transmitted signal field is clearly bunched regardless of which photon-number wave packet impinges onto the slab. Bunching occurs over a time scale of the order of the thermal coherence time, which is now much smaller than the low-temperature case $\tau_{c}$. Similarly, by comparing different absorption regimes, it is seen that in the low-temperature case the correlation times are somewhat shortened by the progressive loss of photon correlations as the pulse traverses a slab with larger absorption. At higher temperatures, increased absorption further inhibits the residual correlations from the pulse component in the transmitted signal yielding a slightly enhanced bunching effect (more chaotic signal).

\section{CONCLUSIONS}

We have used a recently developed quantization scheme for the electromagnetic field in a dispersive and absorbing dielectric slab to determine the effects of transmission through the slab on various nonclassical features of the inci-
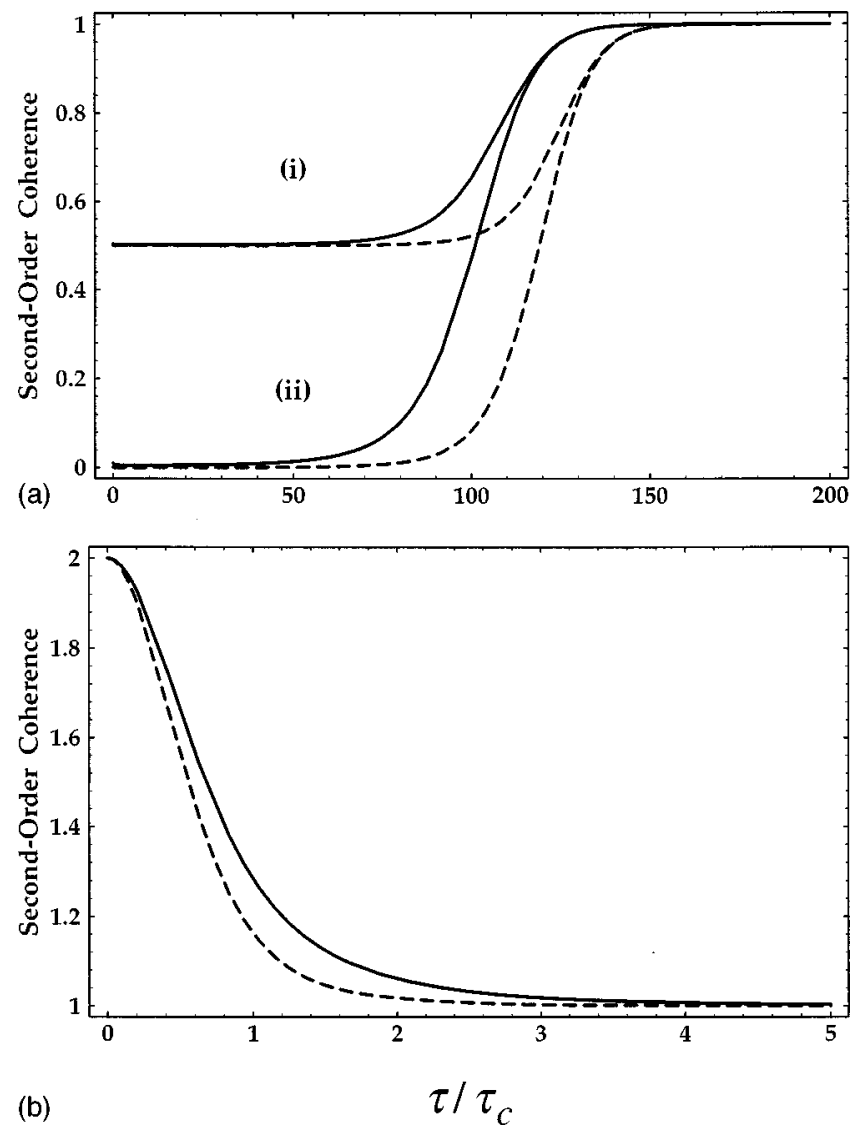

FIG. 5. Degree of second-order coherence (5.13) for a singlephoton and a two-photon Gaussian wave packet that has propagated across a thin slab with extinction coefficient $\kappa_{c}=2.95 \times 10^{-4}$ (dashed curve) and $\kappa_{c}=2.95 \times 10^{-3}$ (solid curve). For all curves, the real refractive index and the magnitude of the transmission coefficient are kept at $\eta_{c}=2.85$ and $\left|T_{c}\right| \cong 0.6$. The pulse carrier frequency $\omega_{c}$ and film thickness $2 l$ are such that $\omega_{c} l / c \cong 500$, while the incident pulse length $L$ is 20 times the film thickness. The two sets of curves (i) and (ii) of part (a) describe, respectively, $g_{2}^{(2)}$ and $g_{1}^{(2)}$ for a slab at temperature $\Theta=2.5 \times 10^{-3} \hbar \omega_{c} / k$. Part (b) refers to a slab at a higher temperature $\Theta=2.5 \times 10^{-1} \hbar \omega_{c} / k$ where the two degrees of coherence essentially coincide. The delay is measured in units of the mean coherence time $\tau_{c}=\hbar / k \Theta$.

dent light beams. Thus for quadrature-squeezed incident light, we have shown how the squeezing of the transmitted light, as measured by balanced homodyne detection, is degraded by the absorption in the slab and by the reflection and transmission effects at the slab surfaces; for slabs at elevated temperatures, the thermal noise generated within the slab produces a further degradation in the squeezing. We have derived a simple relation, Eq. (3.17), for the squeezing in the output from the slab in terms of the squeezings in the two input beams and the thermal noise generated within the slab. We have illustrated these formal results by numerical calculations of the frequency dependence of the squeezing after transmission through a slab in the Reststrahl region of a single dielectric resonance.

Nonclassical aspects of the photon-counting statistics, as measured by direct detection, are also reduced by transmission through the slab. Thus, the Mandel $Q$ factor for incident-squeezed light and the degree of second-order coherence for incident-antibunched light both tend towards 
classical values after transmission, and these tendencies are enhanced when the slab is maintained at an elevated temperature. The frequency variations of the effects of transmission on the Mandel $Q$ factor have also been illustrated by numerical results for the single-resonance form of the dielectric permittivity of the slab. The degree of second-order coherence has been evaluated for the transmission of an incident number-state pulse, and numerical results have been presented for single- and two-photon states. The results obtained for the photon-counting statistics agree with special cases derived previously, but the slab quantization formalism allows a more comprehensive treatment, with proper inclusion of the effects of the surfaces and of thermal generation within the slab.

One could carry out an analogous treatment for a reflection configuration, as opposed to the transmission configuration of Fig. 1. Results similar to the quadrature field fluctuations given by Eq. (3.15) and the Mandel $Q$ factor given by Eq. (4.17) can be derived for the field traveling away from the left of the slab, when the fields $|L\rangle$ and $|R\rangle$ traveling towards the slab retain their natures as a squeezed vacuum and a squeezed coherent state, respectively. These results for the reflection case can be written down directly, simply by interchanging $T\left(\omega_{\mathrm{LO}}\right) \leftrightarrow R\left(\omega_{\mathrm{LO}}\right)$ in Eq. (3.15) and $T\left(\omega_{H}\right) \leftrightarrow R\left(\omega_{H}\right)$ in Eq. (4.17), as expected from the symmetry of the problem. A similar conversion applies to the degree of second-order coherence.

The results derived here for the effects of propagation through a lossy medium on the nonclassical properties of incident light complement those in our previous publication [6], concerned with the quantum-mechanical formulation of the problem, and with the effects of propagation on the initial peak position and width of an incident optical pulse. The predictions for the magnitudes of the latter effects are the same in classical and quantum theories, and they include apparent delay or acceleration and broadening or narrowing of the pulse, depending on its parameters and those of the dielectric medium. The quantum theory thus embraces results derivable by classical theory, where this provides an adequate description of the phenomena, but it also covers the specifically quantum-mechanical effects treated in the present paper, where the classical theory is manifestly inadequate. The thermal noise that inevitably accompanies propagation through an attenuating slab at elevated temperatures is also a quantum-mechanical feature, governed essentially by Planck's law. The one theory thus covers the entire range of phenomena associated with optical propagation through dielectric media.

\section{ACKNOWLEDGMENT}

This work was supported by the European Community Human Capital and Mobility Programme through its network on 'Nonclassical Light', under Contract No. CHRXCT930114.

\section{APPENDIX}

We derive here the expectation values appearing in Eqs. (4.6) and (4.7). The average is over a product state that comprises the incident states of the field traveling leftwards $(|L\rangle)$ and rightwards $(|R\rangle)$ to the slab as well as states $|f\rangle$ that account for losses within the slab (cf. Fig. 1). From the expression (2.3) for the outgoing propagating field one obtains

$$
\begin{aligned}
\left\langle\hat{b}_{R}^{\dagger}(1) \hat{b}_{R}(2)\right\rangle= & R_{1}^{*} R_{2}\left\langle L\left|\hat{b}_{L}^{\dagger}(1) \hat{b}_{L}(2)\right| L\right\rangle \\
& +T_{1}^{*} T_{2}\left\langle R\left|\hat{a}_{R}^{\dagger}(1) \hat{a}_{R}(2)\right| R\right\rangle \\
& +\left\langle f\left|\hat{F}^{\dagger}(1) \hat{F}(2)\right| f\right\rangle
\end{aligned}
$$

and

$$
\begin{aligned}
\left\langle\hat{b}_{R}^{\dagger}(1) \hat{b}_{R}^{\dagger}(2) \hat{b}_{R}(3) \hat{b}_{R}(4)\right\rangle= & \left\langle f\left|\hat{F}^{\dagger}(1) \hat{F}^{\dagger}(2) \hat{F}(3) \hat{F}(4)\right| f\right\rangle+\left\{R_{1}^{*} R_{2}^{*} R_{3} R_{4}\left\langle L\left|\hat{b}_{L}^{\dagger}(1) \hat{b}_{L}^{\dagger}(2) \hat{b}_{L}(3) \hat{b}_{L}(4)\right| L\right\rangle\right. \\
& +R_{1}^{*} R_{2}^{*} T_{3} T_{4}\left\langle L\left|\hat{b}_{L}^{\dagger}(1) \hat{b}_{L}^{\dagger}(2)\right| L\right\rangle\left\langle R\left|\hat{a}_{R}(3) \hat{a}_{R}(4)\right| R\right\rangle+R_{1}^{*} R_{3} T_{2}^{*} T_{4}\left\langle L\left|\hat{b}_{L}^{\dagger}(1) \hat{b}_{L}(3)\right| L\right\rangle \\
& \times\left\langle R\left|\hat{a}_{R}^{\dagger}(2) \hat{a}_{R}(4)\right| R\right\rangle+R_{2}^{*} R_{3} T_{1}^{*} T_{4}\left\langle L\left|\hat{b}_{L}^{\dagger}(2) \hat{b}_{L}(3)\right| L\right\rangle\left\langle R\left|\hat{a}_{R}^{\dagger}(1) \hat{a}_{R}(4)\right| R\right\rangle \\
& +\left\langle f\left|\hat{F}^{\dagger}(1) \hat{F}(3)\right| f\right\rangle T_{2}^{*} T_{4}\left\langle R\left|\hat{a}_{R}^{\dagger}(2) \hat{a}_{R}(4)\right| R\right\rangle+\left\langle f\left|\hat{F}^{\dagger}(1) \hat{F}(4)\right| f\right\rangle T_{2}^{*} T_{3}\left\langle R\left|\hat{a}_{R}^{\dagger}(2) \hat{a}_{R}(3)\right| R\right\rangle \\
& +\left\langle f\left|\hat{F}^{\dagger}(2) \hat{F}(3)\right| f\right\rangle T_{1}^{*} T_{4}\left\langle R\left|\hat{a}_{R}^{\dagger}(1) \hat{a}_{R}(4)\right| R\right\rangle+\left\langle f\left|\hat{F}^{\dagger}(2) \hat{F}(4)\right| f\right\rangle T_{1}^{*} T_{3}\left\langle R\left|\hat{a}_{R}^{\dagger}(1) \hat{a}_{R}(3)\right| R\right\rangle \\
& \left.+\left(R_{i} \leftrightarrow T_{i} \text { and } \hat{b}_{L} \leftrightarrow \hat{a}_{R}\right)\right\},
\end{aligned}
$$

which reduce to

$$
\left\langle f\left|\left\langle 0\left|\left\langle R\left|\hat{b}_{R}^{\dagger}(1) \hat{b}_{R}(2)\right| R\right\rangle\right| 0\right\rangle\right| f\right\rangle=T_{1}^{*} T_{2}^{*}\left\langle R\left|\hat{a}_{R}^{\dagger}(1) \hat{a}_{R}(2)\right| R\right\rangle+\left\langle f\left|\hat{F}^{\dagger}(1) \hat{F}(2)\right| f\right\rangle,
$$




$$
\begin{aligned}
\left\langle f\left|\left\langle 0\left|\left\langle R\left|\hat{b}_{R}^{\dagger}(1) \hat{b}_{R}^{\dagger}(2) \hat{b}_{R}(3) \hat{b}_{R}(4)\right| R\right\rangle\right| 0\right\rangle\right| f\right\rangle= & \left\langle f\left|\hat{F}^{\dagger}(1) \hat{F}^{\dagger}(2) \hat{F}(3) \hat{F}(4)\right| f\right\rangle+T_{1}^{*} T_{2}^{*} T_{3} T_{4} \\
& \times\left\langle R\left|\hat{a}_{R}^{\dagger}(1) \hat{a}_{R}^{\dagger}(2) \hat{a}_{R}(3) \hat{a}_{R}(4)\right| R\right\rangle+\left\langle f\left|\hat{F}^{\dagger}(1) \hat{F}(3)\right| f\right\rangle T_{2}^{*} T_{4} \\
& \times\left\langle R\left|\hat{a}_{R}^{\dagger}\left(\omega_{2}\right) \hat{a}_{R}(4)\right| R\right\rangle+\left\langle f\left|\hat{F}^{\dagger}(1) \hat{F}(4)\right| f\right\rangle T_{2}^{*} T_{3}\left\langle R\left|\hat{a}_{R}^{\dagger}(2) \hat{a}_{R}(3)\right| R\right\rangle \\
& +\left\langle f\left|\hat{F}^{\dagger}(2) \hat{F}(3)\right| f\right\rangle T_{1}^{*} T_{4}\left\langle R\left|\hat{a}_{R}^{\dagger}(1) \hat{a}_{R}(4)\right| R\right\rangle \\
& +\left\langle f\left|\hat{F}^{\dagger}(2) \hat{F}(4)\right| f\right\rangle T_{1}^{*} T_{3}\left\langle R\left|\hat{a}_{R}^{\dagger}(2) \hat{a}_{R}(3)\right| R\right\rangle,
\end{aligned}
$$

when $|L\rangle$ is taken as a conventional vacuum state $|0\rangle$. Here the numbers denote the corresponding frequencies. Notice that in Eq. (A1) the contributions from the transmitted, the reflected, and the slab noise fields are all separate while in the higher-order correlation function (A2) there appear also entangled terms that originate from the interference between these three components.

When the state $|L\rangle$ is a squeezed vacuum and the signal field $|R\rangle$ is in a squeezed coherent state the relevant expectation values on the right-hand side of Eqs. (A1)-(A4) can be evaluated with the help of the results (3.7)-(3.10) and one obtains, after some algebra,

$$
\begin{aligned}
& \left\langle R\left|\hat{a}_{R}^{\dagger}(1) \hat{a}_{R}^{\dagger}(2) \hat{a}_{R}(3) \hat{a}_{R}(4)\right| R\right\rangle=\alpha_{1}^{*} \alpha_{2}^{*} \alpha_{3} \alpha_{4} \\
& +e^{i\left(\varphi_{\sigma_{4}}-\varphi_{\sigma_{1}}\right)} \sinh \sigma_{1} \sinh \sigma_{4}\left\{\delta_{1,2} \delta_{3,4} \cosh \sigma_{2} \cosh \sigma_{3}\right. \\
& \left.+e^{i\left(\varphi_{\sigma_{3}}-\varphi_{\sigma_{2}}\right)}\left[\delta_{1,3} \delta_{2,4}+\delta_{1,4} \delta_{2,3}\right] \sinh \sigma_{2} \sinh \sigma_{3}\right\} \\
& +\alpha_{3} \alpha_{4} e^{-i \varphi_{\sigma_{1}} \delta_{1,2}} \sinh \sigma_{1} \cosh \sigma_{1} \\
& +\alpha_{1}^{*} \alpha_{2}^{*} e^{i \varphi_{\sigma_{3}} \delta_{3,4}} \sinh \sigma_{3} \cosh \sigma_{3}+\alpha_{2}^{*} \alpha_{4} \delta_{1,3} \sinh ^{2} \sigma_{1} \\
& +\alpha_{2}^{*} \alpha_{3} \delta_{1,4} \sinh ^{2} \sigma_{1}+\alpha_{1}^{*} \alpha_{4} \delta_{2,3} \sinh ^{2} \sigma_{2} \\
& +\alpha_{1}^{*} \alpha_{3} \delta_{2,4} \sinh ^{2} \sigma_{2} \text {, } \\
& \left\langle R\left|\hat{a}_{R}(1) \hat{a}_{R}(2)\right| R\right\rangle=\alpha_{1} \alpha_{2}+e^{i \varphi_{\sigma_{1}}} \sinh \sigma_{1} \cosh \sigma_{1} \delta_{1,2},
\end{aligned}
$$

$\left\langle R\left|\hat{a}_{R}^{\dagger}(1) \hat{a}_{R}(2)\right| R\right\rangle=\alpha_{1}^{*} \alpha_{2}+\delta_{1,2} \sinh ^{2} \sigma_{1}$,

$\left\langle L\left|\hat{b}_{L}^{\dagger}(1) \hat{b}_{L}^{\dagger}(2) \hat{b}_{L}(3) \hat{b}_{L}(4)\right| L\right\rangle$

$=e^{i\left(\varphi_{\sigma_{4}}-\varphi_{\sigma_{1}}\right)} \sinh \rho_{1} \sinh \rho_{4}$

$\times\left[\cosh \rho_{2} \cosh \rho_{3} \delta_{1,2} \delta_{3,4}+e^{i\left(\varphi_{\sigma_{3}}-\varphi_{\sigma_{2}}\right)} \sinh \rho_{2} \sinh \rho_{3}\right.$

$$
\left.\times\left(\delta_{1,3} \delta_{2,4}+\delta_{1,4} \delta_{2,3}\right)\right] \text {, }
$$

$\left\langle L\left|\hat{b}_{L}(1) \hat{b}_{L}(2)\right| L\right\rangle=e^{i \varphi_{\sigma_{1}}} \delta_{1,2} \sinh \rho_{1} \cosh \rho_{1}$,

$\left\langle L\left|\hat{b}_{L}^{\dagger}(1) \hat{b}_{L}(2)\right| L\right\rangle=\delta_{1,2} \sinh ^{2} \rho_{1}$.

The noise in the slab originates from the dissipation, described here by states $|f\rangle$ introduced in Sec. II. The fourthorder noise correlation function in Eqs. (A2) and (A4) is expressed in terms of second-order ones as usually done for Gaussian random variables, i.e.,

$$
\begin{aligned}
\left\langle f\left|\hat{F}^{\dagger}(1) \hat{F}^{\dagger}(2) \hat{F}(3) \hat{F}(4)\right| f\right\rangle & \\
= & \left\langle f\left|\hat{F}^{\dagger}(1) \hat{F}(3)\right| f\right\rangle\left\langle f\left|\hat{F}^{\dagger}(2) \hat{F}(4)\right| f\right\rangle \\
& +\left\langle f\left|\hat{F}^{\dagger}(1) \hat{F}(4)\right| f\right\rangle\left\langle f\left|\hat{F}^{\dagger}(2) \hat{F}(3)\right| f\right\rangle
\end{aligned}
$$

that can be evaluated at once by using Eq. (3.14).
[1] S. M. Barnett, R. Matloob, and R. Loudon, J. Mod. Opt. 42, 1165 (1995).

[2] R. Matloob, R. Loudon, S. M. Barnett, and J. Jeffers, Phys. Rev. A 52, 4823 (1995).

[3] T. Gruner and D.-G. Welsch, Phys. Rev. A 53, 1818 (1996).

[4] R. Matloob and R. Loudon, Phys. Rev. A 53, 4567 (1996).

[5] T. Gruner and D.-G. Welsch, Phys. Rev. A 54, 1661 (1996).

[6] M. Artoni and R. Loudon, Phys. Rev. A 55, 1347 (1997).

[7] R. Loudon and P. L. Knight, J. Mod. Opt. 34, 709 (1987).

[8] K. J. Blow, R. Loudon, S. J. D. Phoenix, and T. J. Shepherd, Phys. Rev. A 42, 4102 (1990).

[9] J. R. Jeffers, N. Imoto, and R. Loudon, Phys. Rev. A 47, 3346 (1993).

[10] H. Fearn and R. Loudon, Opt. Commun. 64, 485 (1987).

[11] L. Knöll and U. Leonhardt, J. Mod. Opt. 39, 1253 (1992).
[12] U. Leonhardt, J. Mod. Opt. 40, 1123 (1993).

[13] J. Jeffers and S. M. Barnett, J. Mod. Opt. 41, 1121 (1994).

[14] E. Schmidt, L. Knöll, and D.-G. Welsch, Phys. Rev. A 54, 843 (1996).

[15] L. Mandel, Opt. Lett. 4, 205 (1979).

[16] C. K. Hong, Z. Y. Ou, and L. Mandel, Phys. Rev. Lett. 59, 2044 (1987).

[17] A. M. Steinberg, P. G. Kwiat, and R. Y. Chiao, Phys. Rev. Lett. 71, 708 (1993).

[18] M. S. Kim, L. Allen, and R. Loudon, Phys. Rev. A 50, 3614 (1994).

[19] Similar integrals also appear in problems of specific heats of solids whose treatment is herewith taken up. See, e.g., N. W. Ashcroft and N. D. Mermin, Solid State Physics (Saunders, Philadelphia, 1979), Chap. 24. 